Aus dem Institut für Infektionskrankheiten in Berlin. (Direktor: Geh. Ober-Med.-Rat Prof. Dr. Gaffky. Abteilungsvorsteher: Prof. Dr. Lentz.)

\section{Atypische Bazillenruhr in einer Irren-Heil- und Pflegeanstalt.}

\section{Von Dr. Karl Heuser, Assistenten des Instituts.}

Im Sommer 1908 hatte ich Gelegenheit, anläBlich einer Ruhrepidemie, die in der neuerbauten Provinzial-Heil- und Pflegeanstalt Leubus in Schlesien ausgebrochen war, eine ganze Irrenanstalt auf das Vorhandensein von Ruhrbazillenträgern zu untersuchen.

Die Anstalt Leubus in Schlesien besteht aus einem älteren, kleinen Teile, der in einem früheren Kloster untergebracht ist, und der neuerbauten Anstalt Städtel-Leubus, die etwa $3 \mathrm{~km}$ von dem Kloster entfernt liegt und seit etwa sechs Jahren eröffnet ist.

In dieser neuen Anstalt Städtel-Leubus kamen seit ihrer Eröffnung jährlich vereinzelte Ruhrfälle vor. Im Mai 1908 stieg die Zahl der Erkrankungen innerhalb von drei Wochen auf 24 mit einem Todesfall. Wegen dieser Häufung der Fälle wurde ich seitens des preußischen Kultusministeriums nach Leubus geschickt, um womöglich durch bakteriologische Untersuchungen die Ursache der immer sich wiederholenden Ruhrerkrankungen ausfindig zu machen. Bei meiner Ankunft Mitte Juni war die Epidemie eben wieder erloschen.

Die im Mai an Ruhr Erkrankten waren jedoch noch auf einer Ruhrstation isoliert, wenn sie auch, da seit etwa drei Wochen keine frischen Fälle mehr hinzugekommen waren und die Ruhr bei sämtlichen Kranken milde verlaufen war, bereits als klinisch geheilt angesehen werden konnten. Ihre wiederholte bakteriologische Untersuchung ergab auch in keinem Falle mehr das Vorhandensein von Ruhrbazillen in ihren Faeces. Im Monat Mai waren dagegen die Krankheitserreger von dem Medizinal-Untersuchungsamt in Breslau nachgewiesen und als Ruhrbazillen vom Typus $\mathrm{Y}$ identifiziert worden.

Die 23 Insassen der Ruhrstation gehörten sämtlich der Frauenabteilung an (21 Geisteskranke und 2 erkrankte Pflegerinnen). Auf der Männerabteilung war nur 1 Fall, mit tödlichem Ausgang vorgekommen. Dieser Umstand wies darauf hin, daß durch Lebensmittel, die in der beiden Abteilungen gemeinsamen Anstaltsküche zubereitet wurden, oder durch die gemeinsame Wasserleitung die Seuche nicht hervorgerufen sein konnte. Es blieb also die Wahrscheinlichkeit, daß durch scheinbar Gesunde die Seuche unterhalten wurde. Ich untersuchte deshalb sämtliche Insassen der Anstalt und achtete gleichzeitig auf das Vorkommen von Typhus- und Paratyphusbazillen.

Während der $2 \frac{1}{2}$ Monate, welche die Untersuchung der gesunden Anstaltsinsassen erforderte, kam keine neue Ruhrerkrankung unter ihnen vor. Erst am Tage vor meiner Abreise brach von neuem die Ruhr aus, und zwar in demselben Pavillon der Frauenabteilung, in welchem im Mai d. J. die Epidemie hauptsächlich geherrscht hatte.

Am Tage vor Ausbruch dieser neuen Epidemie hatte ich aus dem diarrhoischen, nur wenig Schleim enthaltenden Stuhl einer Geisteskranken, dio in demselben Pavillon untergebracht war und keine klinischen Ruhrsymptome aufwies, Ruhrbazillen festgestellt. Es lag also nahe, diesen atypischen, klinisch nicht als Ruhr erkannten Fall, der genügend Gelegenheit gab, daß Ruhrbazillen unbemerkt in die Umgebung der Kranken ausgestreut wurden, für die Entstehung dieser neuen, wenn nicht auch der früheren Epidemie im Monat Mai verantwortlich zu machen. Die näheren, später mitgeteilten Umstände scheinen dieser Vermutung Recht zu geben.

Meine neben der bakteriologischen Untersuchung durchgeführten epidemiologischen Feststellungen ergaben außerdem, daf in dem Dorfe Städtel-Leubus mit ungefähr 500 Einwohnern im Monat Mai d. J. etwa 15 Erkrankungen mit ruhrähnlichen Durchfällen, darunter 2 Todesfälle, vorgekommen waren. $\mathrm{Ob}$ auch in anderen Dörfern der Umgegend ähnliche verdächtige Erkrankungen beobachtet worden sind, konnte ich nicht feststellen. Zwischen diesen Dörfern und der Anstalt bestehen nun dadurch besondere Beziehungen, daß einerseits die Pfleger und Pflegerinnen der Anstalt in diesen Orten ihre Familien untergebracht haben, bei denen sie auch zeitweise wohnen; anderseits dadurch, daß harmlose Anstaltspfleglinge in diese Dörfer in Familienpflege gegeben werden.

Hierdurch entsteht genügende Gelegenheit, leichte, klinisch nicht erkannte Ruhrfälle von der Anstalt in die Dörfer und von den Dörfern in die Anstalt zu verpflanzen, sodaß in letzter Linie die Anstalt mit den umgebenden Dörfern als ein einziger Ruhrherd zu betrachten ist.

Die meisten Stühle, mit Ausnahme derjenigen der Rekonvaleszenten der Ruhrstation, konnten aus Mangel an Zeit nur einmal untersucht werden. Dagegen wurde jeder diarrhoische Stuhl wiederholt untersucht.

Während der zuerst vorgenommenen Untersuchung der Frauenabteilung wurde die übliche Untersuchungstechnik angewandt: Ausstreichen der Stühle auf Drigalski-Conradi-Agar, Probeagglutination verdächtiger Kolonien in hochwertigen Ruhrseris; bei positivem Ausfalle Verimpfen der verdächtigen Kolonien in NeutralrotTraubenzucker-Agar, Lackmusmolke, Milch und schließlich makroskopische Agglutination des reingezüchteten Stammes mit hochwertigem künstlichen Ruhrserum bis zu dessen Endtiter.

Es zeigte sich jedoch bald, daß die Darmflora der meisten Anstaltsinsassen zum größten Teil aus einer Coliart bestand, die anf der Drigalski-Conradi-Platte fast blau und zarter als die gewöhnlichen Coliarten wuchs, sodaß eine einzelstehende Ruhrkolonie vom Typus Y, um den es sich hier handelte, zumal auf einer mit Coli dichtbesäten Platte, mit dem Ange kaum herauszufinden war.

Hierzu kam die Tatsache, daß gerade beim Typus Y (HissRussel) häufig schwer agglutinable Stämme angetroffen werden (Lentz), Luksch${ }^{2}$ ), ein Vorkommen, das wir auch vom Typhusbacillus kennen und das ich auch bei frisch gezüchteten Paratyphusbazillen öfter feststellen konnte. Auch bei einem von mir in Leubus frisch aus einem Stuhle gezüchteten Ruhrstamme des Typus Y stellte sich erst nach zwei Wochen, nach wiederholtem Ueberimpfen auf Agar, allmählich eine normale Agglutinabilität ein.

Ich ging deshalb bei Beginn der Untersuchung der Männerabteilung dazu über, nicht mehr den Ausfall der Probeagglutination als ausschlaggebend anzusehen, sondern impfte von jedem Falle möglichst zahlreiche, verdächtige Kolonien auf Schrägagar und in Neutralrot-Traubenzucker-Agar über. Die Stämme, welche sich auf letzterem wie Ruhrbazillen verhielten, wurden dann in üblicher Weise weiter untersucht.

Bei den 23 Ruhrrekonvaleszenten wurden, wie erwähnt, in keinem Falle mehr Ruhrbazillen nachgewiesen. Auch das Blutserum dieser früheren Ruhrkranken agglutinierte nicht mehr die aus dem Berliner Institut mitgebrachten Teststämme des Typus Shiga-Kruse, Flexner und $\mathrm{Y}$ in einer Verdünnung $1: 100$. Wie bereits angegeben wurde, hatte jedoch das Breslauer Medizinal-Untersuchungsamt bei den meisten dieser Fälle während der Epidemie im Monat Mai Ruhrbazillen festgestellt. Es ist also ein schnelles Sinken des Agglutinationstiters bei diesen Ruhrfällen anzunehmen, eine Beobachtung, die auch bei anderen Epidemien von Drigalski-PfuhlSchmiedicke ${ }^{3}$ ), Lentz ${ }^{4}$ ) u. a. gemacht ist.

Die Untersuchung sämtlicher klinisch nicht ruhrkrank gewesenen Insassen der Anstalt, im ganzen etwa 650 Personen, ergab auf der Frauenabteilung zwar in keinem Falle Ruhrbazillen, wohl aber einmal Typhusbazillen in einem gesund aussehenden Stuhle; auf der Männerabteilung dagegen in drei Fällen einen Befund von Ruhrbazillen des Typus X (HissRussel), ebenfalls in gesund aussehenden Stühlen.

Erst als die Anstaltsuntersuchung beinahe abgeschlossen war, erhielt ich zwei Tage vor meiner Abreise jenen bereits erwähnten diarrhoischen, schleimhaltigen Stuhl von der Frauenabteilung, in dem ich ebenfalls Ruhrbazillen des Typus $\mathrm{Y}$ in größerer Zahl nachweisen konnte.

1) Lentz, Ueber die im Sommer 1905 in St. Johann-Saarbrücken beobachtete Ruhrepidemie. Klinisches Jahrbuch 1907, Bd. 17, S. 521. - 2) Luk s ch, Ueber eine Dysenterieepidemie. Wiener klinische Wochenschrift 1906, No.28, S.860. - 3) v. D r i ga $1 \mathrm{ski}$, Pfuhl, S chm iedi cke, Beobachtungen und Untersuchungen über die Ruhr. Verōfentlichungen aus dem Gebiete des Militär-Sanitätswesens 1902, H. 20, S. 65 4) Lentz 1. 
Schließlich wurden bei fünf weiteren, gesunden Insassen der Männerabteilung "ruhrähnliche" Stämme nachgewiesen, die weder mit einem der bekannten Typen des Ruhrbacillus noch untereinander übereinstimmten, sodaß sie für die Aetiologie der früheren Fälle der Anstalt nicht in Betracht kamen.

Die vier von mir gezüchteten Ruhrstämme verhielten sich morphologisch und kulturell vollkommen wie der Typus Y (HissRussel), insbesondere vergoren sie, auf Mannit-, Maltose- und Saccharose-Lackmusagar verimpft, lediglich den Mannit; von einem künstlichen Shiga-Kruse-Serum vom Titer 1:2000 wurden sie höchstens bis zur Verdünnung $1: 100$, von einem Y-Testserum dagegen bis zum Ende und von einem Flexner-Testserum fast bis zum Ende des Titers agglutiniert. Das Blutserum der vier Personen, bei welchen ich Ruhrbazillen fand, agglutinierte in einer Verdünnung von 1:100 weder den eigenen Stamm noch irgend einen der fremden Ruhrstämme.

Bei den drei Fällen mit Ruhrbazillenbefund von der Männerabteilung haben wir es zweifellos mit „Bazillenträgern“ zu tun.

Alle drei Geisteskranken hielten sich schon längere Zeit in der Anstalt auf und weder litten sie zur Zeit der Untersuchung an Ruhr oder einer ruhrähnlichen Krankheit, noch ging aus ihren Personalakten hervor, daß sie etwa vor oder nach der Aufnahme in die Anstalt je eine Ruhr oder eine ruhrähnliche Erkrankung durchgemacht hätten; gleichwohl fanden sich in ihren ganz normal aussehenden Faeces echte Ruhrbazillen, wenn auch nur in spärlicher Zahl. Besonders erwähnenswert ist der eine Fall unter ihnen, namens Kl. Er hatte vor seiner Aufnahme in die Anstalt Leubus (vor zwei Jahren), in einem anderen Krankenhause Typhus durchgemacht, und da nach seiner Aufnahme in die Anstalt Leubus noch monatelang bei ihm Typhusbazillen nachgewiesen wurden, so wurde er seitdem als "Typhusbazillenträger" isoliert gehalten. Bei meiner ersten Untersuchung am 20. Juli wies ich bei ihm auf der Drigalski-Conradi-Platte vereinzelte Ruhrkolonien, jedoch keine Typhusbazillen, auch nicht mit Hilfe des Lentz-Tietzschen Anreicherungsverfahrens mit Malachitgrïnagar nach. Die zweite Untersuchung am 3. August ergab ein völlig negatives Resultat, die dritte und vierte Untersuchung am 7. und 25. August jedoch beidemal wieder nur Rubrbazillen. Das gleichzeitige oder kurz aufeinander folgende Auftreten von Ruhr und Typhus ist in der Literatur bereits wiederholt festgestellt (v. Drigalski, Pfuhl, Schmiedicke ${ }^{1}$ ), Gay und Duval' allerdings wurde in diesen Fällen nicht immer auch der bakterioloxische Nachweis erbracht; in dem Leubuser Falle ist umgekehrt von einer früheren klinischen Ruhrerkrankung nichts bekannt geworden, trotzdem konnten einwandfrei Ruhrbazillen nachgewiesen werden. Der bakteriologische Nachweis der früher festgestellten Typhusbazillen wurde von dem Breslauer Medizinaluntersuchungs. amte geführt.

Ob einer dieser drei Bazillenträger für den Ausbruch der Ruhrepidemie im Mai verantwortlich zu machen sei, konnte nicht mehr festgestellt werden. Am nächsten läge es noch, die Infektion des einzigen auf der Männerabteilung an Ruhr Erkrankten in Zusammenhang mit den dort vorhandenen Bazillenträgern zu bringen. Da indessen von diesen weitere Infektionen auf dieser Abteilung nicht gesetzt zu sein scheinen, so hat die Annahme, daß jener vereinzelt gebliebene Ruhrfall seine Entstehung einer sporadischen Verschleppung von Ruhrkeimen von der Frauenabteilung her verdankt, mindestens ebensoviel Wahrscheinlichkeit für sich.

Bei dem vierten Falle, der zuletzt erkrankten Frau, in deren diarrhoischem Stuhl ich reichliche Ruhrbazillen nachweisen konnte, kann man zweifelhaft sein, ob es sich um eine frische Erkrankung einer bis dahin Gesunden oder um ein Rezidiv bei einer chronischen Bazillenträgerin handelt. Beide Möglichkeiten liegen vor, ohne daß für die eine oder die andere eine größere Wahrscheinlichkeit spräche.

Die Frau war eine Geisteskranke, die im Zustande zunehmender Verblödung unsauber war, sodaß sie mit Kot und Urin "schmierie“. Sie war seit $1 \frac{11}{2}$ Jahren in der Anstalt und hatte klinisch keine Ruhr oder ruhrähnliche Erkrankung durchgemacht. Ihr Stuhl enthielt bei der Untersuchung Anfang August keine makroskopisch erkennbare Blutbeimengung, nur Schleim bei sonst fäkulenter Beschaffenheit; auch die ubrigen Krankheitserscheinungen waren so

1) v. Drigalski, Pfuhl, Schmiedicke l. c. - 2) Gay and Duval, Acute dysentery associated with the two types of Bac. dysenteriae Shiga. Pennsylv med. Bulletin 1903. - 3) Ni et e $\mathrm{r}$ und $\mathrm{L}$ i e $\mathrm{f} \mathrm{m}$ a $\mathrm{n} \mathrm{n}$, Ueber bemerkenswerte Befunde bei Untersuchung auf das Vorhandensein von Typhusbazillenträgern in einer Irrenanstalt. Münchener medizinische Wochenschrift 1906, S. 1611. leicht, daß ohne bakteriologische Untersuchung aus dem klinischen Befunde allein die Ruhr nicht hätte diagnostiziert werden können. Die erwähnten Verhältnisse erklären im Verein mit dem Umstand, daß die Frau in ihrem Stuhlgang große Mengen von Ruhrbazillen ausschied, zur Genüge, daß sie, ehe sie durch Isolierung unschädlich gemacht werden konnte, bereits mehrere Saalgenossen infiziert hatte, sodaß am Tage nach Sicherung der positiven bakteriologischen Ruhrdiagnose in demselben Pavillon wiederum eine kleine Ruhrepidemie ausbrach.

Ob sie auch die Epidemie im Monat Mai veranlaßt hat, muß dahingestellt bleiben. Ich hatte den Stuhl derselben Kranken bereits Anfang Juli im Laufe der Untersuchung der Frauenabteilung mit negativem Resultate untersucht; der Stuhl zeigte damals makroskopisch keine abnorme Beschaffenheit. Es wäre aber moglich, daß die betreffende Kranke bereits im Monat Mai d. J., zur Zeit der ersten Epidemie, einen atypischen Anfall hatte, dann Anfang Juli zur Zeit der ersten Untersuchung normal aussehenden Stuhl aufwies und um Anfang September ein Rezidiv bekam. Die Entscheidung darüber, ob es sich bei dieser Kranken um chronische Ruhrprozesse, $d . h$. atonische Darmgeschwüre handelte, hätte am ehesten nachträglich durch das Rektoskop getroffen werden können, wie es von $L e n t z^{1}$ ) und $K$ üster ${ }^{2}$ ) empfohlen ist; doch mußte auf diese Untersuchung bei der Geisteskranken verzichtet werden.

Immerhin beweist der eben geschilderte Fall von neuem, unter wie leichten Symptomen die Ruhr verlaufen kann, und läßt die Mahnung Kruses ${ }^{3}$ ) gerechtfertigt erscheinen, dab jede Erkrankung an Diarrhoe mit Beimengung von Schleim in den Faeces als ruhrverdächtig zu behandeln und der bakteriologischen Untersuchung zu unterwerfen ist.

Bei anderen Infektionskrankheiten, z. B. Cholera, Typhus, Diphtherie spielen die "atypischen" Erkrankungen eine bereits anerkannte Rolle. Bei der Ruhr jedoch hat die atypische Erkrankungsform trotz ihrer epidemiologischen Bedeutung noch nicht die genügende Beachtung gefunden. Und doch müßte bei ihr ebenso wie bei der Diphtherie, bei der in Epidemiezeiten die bakteriologische Untersuchung jeder Halsentzündung schon ganz allgemein gefordert wird, ebenfalls an Orten, an denen die Ruhr nicht schwindet (z. B. Pflegeananstalten, Heerlagern etc.), und in Epidemiezeiten die bakteriologische Untersuchung jedes Diarrhoefalles vorgenommen werden.

Schlußsätze. Bei der einmaligen Durchuntersuchung der Anstalt Städtel-Leubus auf Ruhrbazillen wurden drei „Ruhrbazillenträger" und eine "atypische Ruhrdiarrhoe" festgestellt. An diese letztere schloB sich eine kleine Ruhrepidemie in demselben Pavillon der Anstalt, in welchem die betreffende Diarrhoekranke untergebracht war. Die "atypische Ruhrdiarrhoe" hat in der Literatur bisher nur geringe Beachtung gefunden. Angesichts des Umstandes jedoch, dab an atypischer Ruhrdiarrhoo Leidende reichliche Ruhrbazillen in ihren Faeces ausscheiden, die klinischen Ruhrsymptome dagegen bei dieser Krankheits. form nur sehr wenig ausgeprägt sind, muß die Forderung aufgestellt werden, da@ ähnlich wie in Diphtheriezeiten die bakteriologische Untersuchung jeder Halsentzündung, auch an Orten, an denen die Ruhr nicht schwindet, z. B. Pflegeanstalten, Heerlagern etc., sowie in Epidemiezeiten die bakteriologische Untersuchung jeder Diarrhoe vorgenommen wird. In Leubus erstreckte sich die Ruhr nicht allein auf die Anstalt, sondern auch auf die benachbarte Ortschaft, in der die Pfleger und Pflegerinnen ihre Familien untergebracht haben, in der sie daher zeitweise selbst wohnen und in der harmlose Geisteskranke der Anstalt in Familienpflege untergebracht werden. Außerdem stehen die Anstalten derselben Provinz, in denen fast ständig vereinzelte Fälle von Ruhr und Typhus vorkommen, in einem fast täglichen Austausch ihrer Geisteskranken. Es ist daher fast täglich die Möglichkeit gegeben, daß neue, klinisch nicht erkannte Fälle von Ruhr und Typhus in Gestalt von Bazillenträgern und von leichten atypischen Erkrankungen in eine solche Anstalt eingeführt werden. Eine dauernde $\mathrm{Be}$ freiung einer solchen Anstalt von diesen Seuchen ist somit nur von einer dauernden bakteriologischen Kontrolle jeder Neuaufnahme sowie des Pflegepersonals zu erwarten.

1) Lentz , Diskussionsbemerkung. Zentralblatt für Bakteriologie, l. Abteilung, Ref. Beiheft zu Bd. 42, S. 6l. - 2) K üster, Ein Dysenteriebazillenträger. Münchener medizinische Wochenschrift 1908, S. 1833 - 3) K r u s e. Die Verbreitung der Ruhr durch sogenannte "Datierausscheider" und "Bazillenträger". Klinisches Jahrbuch 1908, Bd. 19, S. 529 